\title{
Application of Transient Energy Function in Regional Power Grig
}

\author{
Tang Meng-meng ${ }^{1,}$, Wang Bo ${ }^{2, b}$, Liu Di-chen ${ }^{3, c}$ and He Zhong-wei ${ }^{4, d}$ \\ School of Electrical Engineering, Wuhan University, Wuhan, China \\ aTangmengmeng2008@163.com, ${ }^{\mathrm{b}}$ whwdwb@gmail.com \\ cdcliu@whu.edu.cn, ${ }^{\mathrm{c}} \mathrm{hzw19860727@126.com}$
}

Keywords: regional system; transient energy function; stable equilibrium

\begin{abstract}
With expanding scale of modern power system and cross-region connection of power grid, the system often run closer to the ultimate state. So the security and stability problems become a focus of power system. This paper proposes an accurate regional-equivalent-system model, and the transient energy function is constructed based on this model, using above energy function the transient energy and critical energy can be calculated, and the units' stability can be determined. Finally this method is applied to 8-machine-36-node system. The validity is confirmed by PSASP simulation.
\end{abstract}

\section{Introduction}

In the power system, the synchronous generators keep synchro -speed is a necessary condition of the normal operation, if it can't make the generator to keep each other or not recover synchronous operation after losing synchro momentarily, which makes the electric power system to lose stability ${ }^{[1]}$. The destruction of the power system stability, often can cause disconnection and collapse of the system, which caused a big power outages, so power system stability should be insured. According to the statistics, the damages of transient stability rate to the fore, so transient stability analysis become the subject of dynamic security assessment.

There have two main methods for transient stability analysis. One is time dominated simulation method, it has high precision and can calculate under the complex system model, but can't give stability margin and its calculation speed is too slow, so it only is an off-line method. Another is direct method. Energy function method is the one important direct method. Its advantages lay in that it can give stability margin and has fast calculation speed. In recent years, These studies improve its precision, make it basically satisfy the demands of power system ${ }^{[2]}$. It can be a fast on-line scanning method for transient stability.

At present the recognized transient energy function methods mainly have leading unstable balance points based on the stability boundary method (CUEP), potential energy boundary surface method ( $P E B S$ ), extended equal area criteria method( EEAC), mixing method.

Among them EEAC method divide the fault machine into group and equivalent them to one machine - infinity bus(OMIB) and use Equal Area Criterion to judge stability ${ }^{[3]}$. Prominent advantages of this method is fast computing, and it avoids the construction of energy function of multi-machine system. But it dependents on the correct identification of critical machines, and it will have greater error when the coherence of unit is bad. So through improvement of the model precision and homology fleet identification, the EEAC method can be further researched in this direction.

This paper proposes a practicable dynamic model for power system transient stability analysis based on energy function, which possesses satisfied accuracy. Through the equivalence of two regions, a 2-machine system is built and utilizing rotor acceleration equation an equivalent single machine infinitive bus system is achieved. Based on this model, equivalent transient energy function is constructed to calculate critical energy after the new stable equilibrium point is solved. System stability can be judged by two manners, namely the comparison of transient energy with critical energy and stability region. 


\section{Regional-equivalent Model}

The premise of application energy function methods for transient stability analysis is structure, or define a transient energy function which can reflect the stability of the practical system, but transient energy function has not a unified structure method, which can only structure different transient energy functions according to different system model ${ }^{[4]}$. This paper proposes a equivalent model which simplified the regional system , equivalent method are described below.

\section{Equivalence of Load}

Consider the interconnected system of the analytical area , the diagram of its power flow balance as shown in figure 1 below.

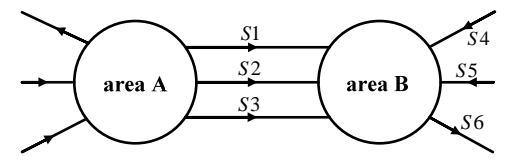

Fig. 1. Power flow balance diagram

The power dynamic characteristics of Regional lines (such as lines 4, 5and 6) may real-time reflect the influence of the external system to the $\mathrm{B}$ area system. After the following handling can get the equivalent load of area B (area A similar) :

(1) Dividing observed section, and selecting the line of the highest voltage sensitivity as observation line, as the interconnection of equivalent area system (such as line 1).

(2) The lines which connected with B system can be equivalent to the power supply or load, among them the interconnections, which power flow inflow area $\mathrm{B}$, will be equivalent to the power supply (such as line 1, 2, 3, 4, and 5); which power flow outflow area B, will be equivalent to the load (such as line 6).

(3) In the equivalent load the corresponding parts should be deducted and added, another lines without the line of the (1) selected, should be broken(i.e. line 1 is reserved). According to the assumptive flow direction of figure 1 , the equivalent load of $\mathrm{B}$ area is:

$S_{B(E Q)}=S_{L}-S_{1}-S_{2}-S_{3}-S_{4}-S_{5}+S_{6}$

Using the same method can get the equivalent load of A area $S_{A(E Q)}$

\section{Equivalence of Synchronous Generators}

Using the real-time voltage on both ends of the observed line, the real-time transient electric potential of the equivalent generator can be obtained, which indicate the dynamic characteristics of regional generator.

(1)The weighted polymerization is adopted to calculate inertia time constant $T_{\text {Jeq }}$ of equivalent synchronous generator and equivalent transient reactance $X_{d}{ }^{\prime}$. The inertia time constant value $M_{N i}$ is converted per-unit value based on the reference value $S_{B}: M_{i}=M_{N i}\left(S_{N i} / S_{B}\right), S_{N i}$ is the rated capacity of each sets itself(i=1,2, ...,n). The equivalent time constant inertia ${ }^{[5]}$ is

$$
M_{e q}=\sum_{i=1}^{n} M_{i}
$$

Equivalent transient reactance is

$$
X_{d}{ }^{\prime}=\left(\sum_{i=1}^{n} X_{d i}^{-1}\right)^{-1}
$$

in which $X_{d i}$ is the transient reactance of the Ith synchronous machine . Above parameters are constants, the output can be queried in PSASP foundation database.

(2) Using the port potential $\dot{U}$ of observed line, the transient potential $E$ ' of equivalent machine can be got according to type (4)

$$
E^{\prime}=\dot{U}+j I_{G} X_{d}^{\prime}
$$

in which $I_{G}=I_{L O A D}+I_{L I N E}$ is the dynamic value of current, as shown in figure 3, $X_{d}$ ' is equivalent transient reactance, (1) has been really. 
Although the actual system may have more generator, but as long as the synchronous generator keep basic synchro, it could be equivalent to a machine, and nothing to do with the number of specific generator sets ${ }^{[6]}$.

\section{System Structure}

Observed line adopt $\pi$ type equivalence after A and B area in figure 1 were equivalent according to the above method, then equivalent model will be got, as shown in figure 2 below.

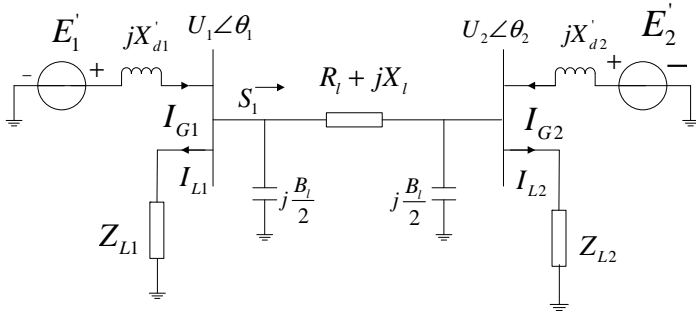

Fig.2. The structure of equivalent system

\section{Transient Energy Function Based on the System of Wide-area Equivalent}

Transient stability analysis research the swing characteristics of generator rotor, which has to do with the power frequency component of network, so the generator can adopt practical model ignored the transient of the stator ${ }^{[7-9]}$. Putting the voltage source in series in networks at the generator inner nodes, generator node extends to inner potential node, transient reactance become a part of the transmission network, load is jointed into the load node in the form of parallel grounding branch, through above steps the admittance matrix can be obtained, which shrinks to the generator inner nodes :

$$
Y=\left[\begin{array}{ll}
Y_{11} & Y_{12} \\
Y_{21} & Y_{22}
\end{array}\right]
$$

Generator rotor motion equation:

$M_{1} \ddot{\delta}_{1}=P_{M 1}-P_{E 1}$

$M_{2} \ddot{\delta}_{2}=P_{M 2}-P_{E 2}$

$P_{M 1} 、 P_{M 2}$ is mechanical power of generator 1, 2. $P_{E 1} 、 P_{E 2}$ is Electromagnetic power of generator $1,2$.

Supposing $\delta=\delta_{1}-\delta_{2}$ as rotor Angle relative of 2 machines, from (6) and (7), we can get:

$$
\begin{aligned}
\ddot{\delta} & =\ddot{\delta}_{1}-\ddot{\delta}_{2} \\
& =\left(P_{M 1}-P_{E 1}\right) / M_{1}-\left(P_{M 2}-P_{E 2}\right) / M_{2}
\end{aligned}
$$

Using $M_{e q}=M_{1} M_{2} /\left(M_{1}+M_{2}\right)$ multiplied by both ends of (8), relative acceleration equations will be got as follow:

$M_{e q} \ddot{\delta}=P_{M}-P_{E}$

Where, $P_{M}=\left(M_{2} P_{M 1}-M_{1} P_{M 2}\right) /\left(M_{1}+M_{2}\right), P_{E}=\left(M_{2} P_{E 1}-M_{1} P_{E 2}\right) /\left(M_{1}+M_{2}\right)$

Mechanical power of generator is given as

$$
\begin{aligned}
& P_{E 1}=E_{1}^{2} G_{11}+E_{1} E_{2} Y_{12} \cos \left(\phi_{12}-\left(\delta_{1}-\delta_{2}\right)\right) \\
& P_{E 2}=E_{2}^{2} G_{22}+E_{1} E_{2} Y_{12} \cos \left(\phi_{21}-\left(\delta_{1}-\delta_{2}\right)\right)
\end{aligned}
$$

Where, $E_{i}=E_{i} \angle \delta_{i}, \quad Y_{i j}=G_{i j}+B_{i j}=Y_{i j} \angle \phi_{i j}, \mathrm{i}=1,2$

Put (10) and (11) substitute into (8), Simplified form is given as

$$
P_{E}=P_{E}^{\prime}+P_{e m} \sin (\delta-\gamma)
$$

Where $P_{E}^{\prime}=\left(M_{2} E_{1}^{2} G_{11}-M_{1} E_{2}^{2} G_{22}\right) /\left(M_{1}+M_{2}\right)$

$$
P_{e m}=\frac{E_{1} E_{2} Y_{12}}{M_{1}+M_{2}} \sqrt{M_{1}^{2}+M_{2}^{2}-2 M_{1} M_{2} \cos 2 \phi_{12}}
$$




$$
\gamma=-\operatorname{ctg}^{-1}\left(\frac{M_{1}-M_{2}}{M_{1}+M_{2}} \operatorname{ctg}_{12}\right)-90^{\circ}
$$

Put(12),(13) and (14) substitute into (9), the rotor motion equation of equivalent one machine-infinity bus is given as

$$
M_{e q} \ddot{\delta}=P_{M}-\left(P_{E}^{\prime}+P_{e m} \sin (\delta-\gamma)\right)
$$

Put (16) to write in the form after failure

$$
M_{e q} \ddot{\delta}=P_{M 3}^{\prime}-P_{e m 3} \sin \left(\delta^{\prime}\right)
$$

Where, $P_{M}^{\prime}=P_{M}-P_{E 3}^{\prime}, \delta^{\prime}=\delta-\gamma$

For the system after failure, set $\delta_{s}{ }^{\prime}$ and $\delta_{u}{ }^{\prime}$ as the stable equilibrium point and the unstable equilibrium point of (17),so $\delta_{s}^{\prime}=\arcsin \left(P_{M 3}^{\prime} / P_{e m 3}\right), \delta_{u}^{\prime}=\pi-\delta_{s}^{\prime}$. The energy function of the system after failure can be expressed as transient energy

$$
\begin{aligned}
V_{c}= & \frac{1}{2} M_{e q} \ddot{\delta}-\left(P_{M}-P_{E 3}^{\prime}\right)\left(\delta-\delta_{s}{ }^{\prime}-\gamma\right) \\
& -P_{e m}\left[\cos (\delta-\gamma)-\cos \left(\delta_{s}{ }^{\prime}\right)\right]
\end{aligned}
$$

Critical energy $V_{c r}=P_{e m}\left[2 \cos \left(\delta_{s}{ }^{\prime}\right)-\left(\pi-2 \delta_{s}{ }^{\prime}\right) \sin \left(\delta_{s}{ }^{\prime}\right)\right]$

\section{Simulation Examples}

\section{Boundary Conditions}

This paper chooses the system of WEPRI8 machine 36 nodes as the research object, and makes the simulation analysis. divide sections by the dotted line (as shown in figure 3), and select the line (BUS22-BUS21) of sections as observation line, equivalent the other parts , and structural transient energy function, finally use PSASP6.28 simulation program and MATLAB programming $^{[10-11]}$ for analysis.(benchmark capacity $S_{B}=100$ MVA)

Fault is set as

(1) Set a three-phase short-circuit at ground fault for $1 \mathrm{~s}-1.1 \mathrm{~s}$ near the BUS23 side between BUS22 and BUS23.

(2) Set a three-phase short-circuit at ground fault for $1 \mathrm{~s}-1.3 \mathrm{~s}$ near the BUS9 side between BUS9 and BUS23.

\section{Example Analysis}

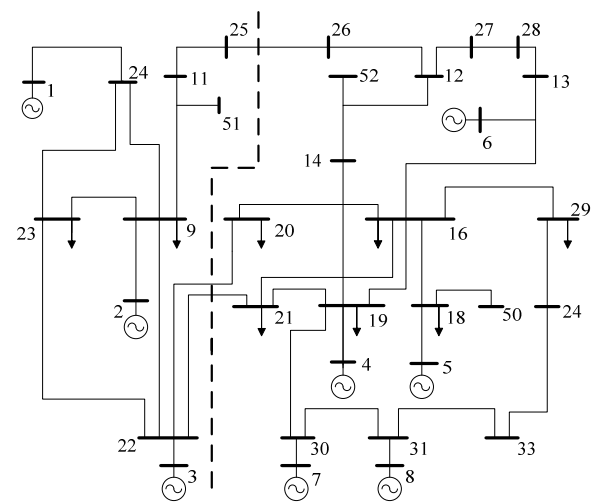

Fig.3. 8 machine 36 node system wiring figure

This paper analyses the fault by comparing the time domain simulation with transient energy function curve based on the wide-area equivalent model, the two methods of fault analysis. Curve as shown in figure 4 (a), figure 4 (b), figure 5 (a), figure 5 (a) below. 


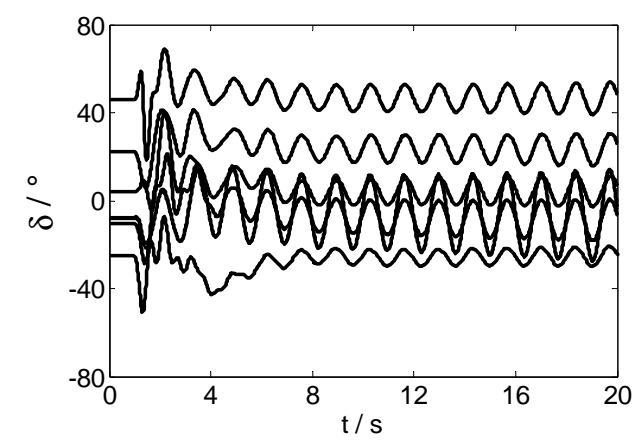

Fig.4. (a) Relative power-angle curve when three-phase short-circuit at $0.1 \mathrm{~s}$

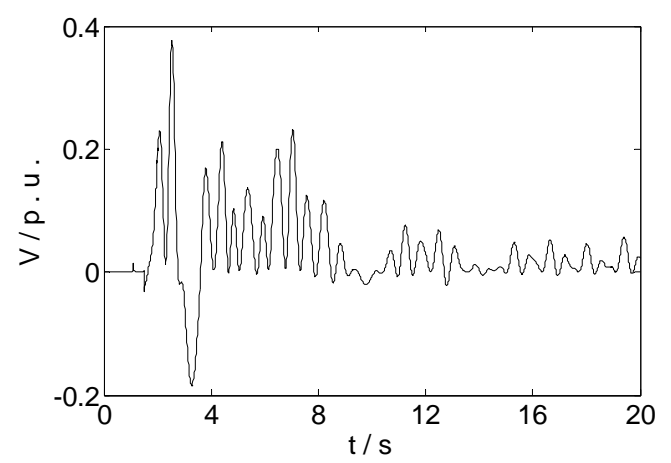

Fig.4. (b) The transient energy function curve when three-phase short-circuit at $0.1 \mathrm{~s}$

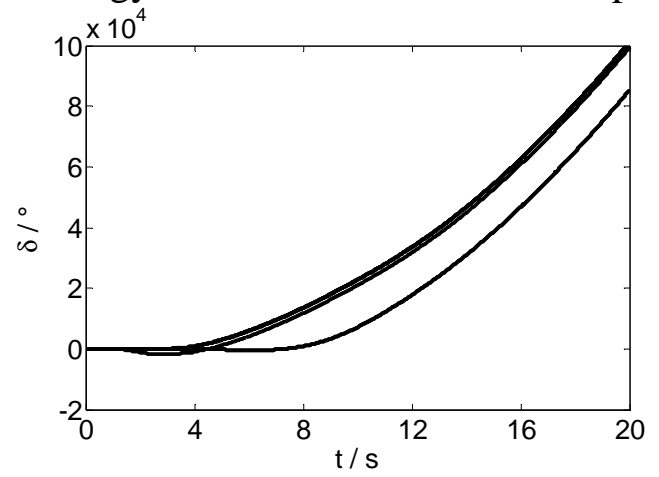

Fig.5. (a) Relative power-angle curve when three-phase short-circuit at $0.3 \mathrm{~s}$

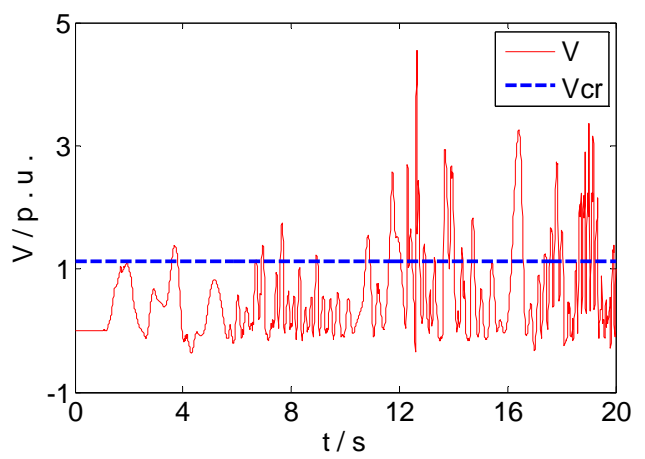

Fig.5. (b) The transient energy function curve when three-phase short-circuit at $0.3 \mathrm{~s}$

For the fault (1), transient energy $\mathrm{V}$ in fig.4. (b) began to accumulate from the instant of failure , increase gradually, which is smaller because of small disturbance. Transient energy begins to be absorbed by system after removing failure, so the equivalent system become stable gradually after disturbance is cut. For fault (2) critical energy was 1.133 p.u.,fig.5. (b) puts critical energy (linear) together with transient energy, the change of system energy can be observed directly observation. From the fig. 5. (a) can be seen, relative power-angle began to diverge in about $4 \mathrm{~s}$ and the system began losing stability, while in fig.5. (b) the energy function may gain the same results, corresponding relation is clear. 


\section{Conclusion}

The analysis of power system transient stability investigate the ability whether each dynamo keep synchronous operation or not when the system suddenly undergo a big disturbance ${ }^{[12]}$, which has very important significance for the safe and reliable operation of the power system. This paper studies the application of transient energy function method in the transient stability analysis, which derives equivalent model of one machine - infinity bus in transient stability analysis based on the model of the area system, and which make analysis on the 8 machine 36 nodes system ,its results in accordance with the results of PSASP program, so that verify the validity of equivalent method and the model.

\section{Acknowledgment}

Supported by:

National High Technology Research and Development Program 863(No: 2011AA05A119)

State Grid Corporation of China, Major Projects on Planning and Operation Control of Large Scale Grid(SGCC-MPLG029-2012)

\section{References}

[1] YU Yi-xin,, CHEN Li-yi. Security and Stability of Electric power System [M]. Science press. 1988.7.

[2] PRABHA KUNDUR. Power System Stability and Control [M]. Beijing: China power press, 2002, 5.

[3] LIU Sheng, WANG Jing. The Energy Function Analysis of Power System Transient Stability [M]. Shanghai: Shanghai JiaoTong University Press, 1996.4.

[4] Wan Qiu-lan,Shan Yuan-da.Evaluation of The Transient Energy Function Methods for Power System Transient Stability Analysis [J] .Automation of Electric Power Systems,2001,25(6):57-59

[5] Maria G A,Tang C,Kim J.Hybrid Transient Stability Analysis[J].IEEE Trans on Power Systems,1990,5(2):384-393.

[6] LIU Sheng.Transient Energy Function Analysis for Power System Stability[J].Power System Technology,1995,19(2):11-17

[7] Ishigame A,Taniguchi T.Transient Stability Analysis for Power System Using Lyapunov Function with Load Characteristics[C]//IEEE Power Engineering Society 2003 General Meeting. New Jersey:IEEE Press,2003:13-17.

[8] Zhang Hong-bin,Li Li,He Ren-mu.Application of Dynamical and Static Load in Transient Stability calculations[J] . Electric Power Automation Equipment,2003,23(6):49-53

[9] Wu Hong-bin,Ding Ming,Li Sheng-hul.Probabilistic Analysis on Influence of Generator Model and Load Model on Transient Stability[J].Power System Technology,2004,28(1):19-21,47

[10] Fu Hong-jun,Ju Ping,Sun Ran,et al.Contrast Studies on Load Modeling of Wide Area Power Grid [J].Automation of Electric Power Systems,2010,34(5):43-47

[11] Zhang Hong-bin,Tang Yong,Li Bai-qing.Study on Dispersing of Difference Equation Load Model Parameters[J].Proceedings of the CSEE,2006,26(18):1-5

[12] Wang Ying,Tan Ding-zhong,Wang Xiao-ying,et a1.Application of Risk Based Transient Stability and Security Assessment in Power System[J].Power System Technology,2003, 27(12): $37-41$ 